\title{
Evaluation of Three-Dimensional Conformal Radiotherapy and Intensity Modulated Radiotherapy Techniques for Left Breast Post-Mastectomy Patients: Our Experience in Nigerian Sovereign Investment Authority-Lagos University Teaching Hospital Cancer Center, South-West Nigeria
}

(D) Samuel Adeneye $e^{1}$, (D) Michael Akpochafor ${ }^{1}$, (1) Bolanle Adegboyega ${ }^{2}$, (1) Adewumi Alabi ${ }^{1}$, (D) Nusirat Adedewe ${ }^{1}$,

(1) Adedayo Joseph ${ }^{3}$, (1) Omolara Fatiregun ${ }^{4}$, (1) Akintayo Omojola , (1) Abe Adebayo², (1) Esther Oluwadara ${ }^{1}$

${ }^{1}$ Department of Radiation Biology, Division of Radiotherapy and Radiodiagnosis, College of Medicine, University of Lagos/NSIA-LUTH Cancer Centre, Lagos, Nigeria

${ }^{2}$ Department of Radiotherapy, Lagos University Teaching Hospital, Lagos, Nigeria

${ }^{3}$ Department of Radiotherapy, Nigeria Sovereign Investment Authority-Lagos University Teaching Hospital, Lagos, Nigeria

${ }^{4}$ Medical Physics Unit, Federal Medical Centre Asaba, Asaba, Nigeria

${ }^{5}$ Department of Radiology, Lagos State University College of Medicine, Lagos, Nigeria

\section{ABSTRACT}

Objective: This study aimed to evaluate the dosimetric properties of treatment plans obtained from three-dimensional conformal radiotherapy (3D-CRT) and intensity-modulated radiotherapy techniques (IMRT) plans for left chest wall breast cancer patients.

Materials and Methods: A total of 20 patients with left-sided chest wall radiotherapy were randomly selected with the dose prescriptions: 42 Gy and $45 \mathrm{~Gy}$ in 15 and 18 fractions, respectively. Treatment plans were obtained using 3D-CRT and IMRT for each patient. Five to seven beams were used for IMRT, while tangential beams were used for 3D-CRT. Planning target volume, $\mathrm{D}_{\text {near-max }}\left(D_{2}\right), \mathrm{D}_{\text {near-min }}\left(D_{98}\right), \mathrm{D}_{\text {mean }}$, Homogeneity and Conformity Indices ( $\mathrm{HI}$ and $\mathrm{CI}$ ) were obtained. Similarly, mean doses to organs at risk (OAR), $\mathrm{V}_{5}, \mathrm{~V}_{10}, \mathrm{~V}_{20}, \mathrm{~V}_{25}$ were generated from the dose-volume histogram and compared. Results: IMRT showed a significant improvement in HI compared to 3D-CRT ( $<<0.0001)$. Although there was no significant difference in sparing of the left lung between both plans for high-dose volumes (V20: 18.2 vs 30.55, p $<0.0001)$, (V25: 11.17 vs 28.12, $\mathrm{p}<0.0001$ ). IMRT however showed supremacy to 3D-CRT with high-dose volumes for the heart, including V20 (4.44 vs 10.29, $\mathrm{p}=0.02)$, V25 (2.08 vs 8.94, $\mathrm{p}=0.002)$. 3D-CRT was better than IMRT in low-dose volumes for left lung (V5: 92.23 vs 56.60, p<0.001; V10: 60.98 vs 47.20, p = 0.04) and heart (V5: 57.45 vs 30.39, $p=0.004)$.

Conclusion: IMRT showed better homogeneity and sparing of high-dose volumes to OAR than 3D-CRT. On the other hand, 3D-CRT showed a reduction of low-dose volumes to OARs than IMRT.

Keywords: IMRT, 3D-CRT, PMRT, organs at risk, radiotherapy

Cite this article as: Adeneye S, Akpochafor M, Adegboyega B, Alabi A, Adedewe N, Joseph A, Fatiregun O, Omojola A, Adebayo A, Oluwadara E. Evaluation of Three-Dimensional Conformal Radiotherapy and Intensity Modulated Radiotherapy Techniques for Left Breast Post-Mastectomy Patients: Our Experience in Nigerian Sovereign Investment Authority-Lagos University Teaching Hospital Cancer Center, South-West Nigeria. Eur J Breast Health 2021; 17(3): 247-252

\section{Key Points}

- $\quad$ The dosimetric properties of 3D-CRT and IMRT for left-chestwall in breast cancer patients were evaluated on 20 patients.

- Dosimetric paramaters of PTV and OARs were obtained and analyzed from the DVH.

- $\quad \mathrm{HI}$ and CI were also calculated and compared.

- $\quad$ Although, HI was better in IMRT than 3D-CRT, showed better sparing of low-dose volumes to OARs. 


\section{Introduction}

Breast cancer is the most frequently diagnosed life-threatening malignancy that affects women, and the leading cause of cancer death in women globally (1). It accounts for $15.3 \%$ of cancer diagnosed globally and $7 \%$ of cancer-related deaths (2). Unlike in developing countries, the survival rate of breast cancer has increased in developed countries over the past 20 years, and more women are now being treated successfully than in previous years. The majority of patients in developing countries present an advanced stage of the disease, owing to several factors that include late presentations, delay in making an appropriate diagnosis, lack of access to cancer treatment, unavailability of advanced technology and infrastructure, poor health practices, ignorance, poverty, and several others (3). Breast cancer treatment is multifactorial-dependent, involving surgery, chemotherapy, radiotherapy, hormonal therapy, and targeted therapy. Over the years, advances in technology have helped to improve the survival rate.

Moreover, newer technologies and treatment methods are currently being developed. Radiotherapy plays a significant role in preventing local and regional recurrence in breast cancer care. It is an important aspect of breast cancer treatment, which minimizes the risk of regional recurrence and enhances the overall life of early-stage breast cancer and the locally advanced disease following mastectomy $(4,5)$. However, chest wall and regional lymph node irradiation is one of the most complex challenging strategies of radiation. Radiotherapy has a significant impact in the management of breast cancer. Radiating the chest wall and regional lymph nodes, such as supraclavicular, axillary, and internal mammary nodes, requires special care for lung, cardiac, and contralateral breast doses. The main aim of radiotherapy is to treat the target volume and protect the surrounding healthy tissues. Three-dimensional conformal radiotherapy (3D-CRT) and intensity-modulated radiotherapy (IMRT) are most often used in the treatment of breast cancer. IMRT is a form of 3D-CRT that further modifies the radiation beam, varying the intensity of radiation to allow optimal treatment precision and dose delivery. It directs radiation at the postmastectomy tumor bed and modulates the intensity of the radiation beams with laser accuracy, thus ensuring the sparing of surrounding healthy tissues (6). This study was conducted at our cancer center to compare the dosimetric properties in 3D-CRT and IMRT on patients with left post-mastectomy breast cancer who received chest wall radiation.

\section{Materials and Methods}

\section{Patients}

Between June 2019 and September 2020, a total of 20 women (aged over 18 years) with invasive ductal carcinoma (T0-T3, N0-N1), leftsided breast cancer treated with modified radical mastectomy, followed by radiotherapy to the chest wall, axillary lymph nodes levels I-III, and supraclavicular fields were randomly selected at the NSIA-LUTH Cancer center, Lagos, Nigeria. All patients were treated with IMRT. Chest walls, including supraclavicular and axillary lymph nodes, were re-planned using 3D-CRT on the Varian's eclipse treatment planning system (TPS) version 15.6 (7).

\section{Computed tomography simulation}

Patients were scanned with 16-slice General electric computed breast board, CT Simulation was performed in a supine position, with arms raised and head in a fixed position. Images were obtained in digital imaging and communication in medicine format and transferred to the Eclipse TPS version 15.6 (VARIAN medical systems) (7).

\section{Target definition}

Under the protocol of the International Commission on Radiation Units and Measurements (ICRU report 83, 2010), all volumes, gross tumor volume, clinical target volume (CTV), planning target volume (PTV), heart, ipsilateral and contralateral lung, contralateral breast, and whole body were delineated. The PTV was restricted to the chest wall, nodes, and supraclavicular, which encloses the CTV with a $1 \mathrm{~mm}$ margin. All patients were treated with the Varian Linear accelerator (Varian Medical System, Palo Alto, CA, USA), which utilizes the Eclipse TPS with $6 \mathrm{MV}$ and $10 \mathrm{MV}$ photon beam. Dose and fractionation for all targets were between 42 Gy and 45 Gy for 15 and 18 fractions, respectively. The goal of each plan was to deliver $95 \%$ of the prescribed dose to $100 \%$ of the target volume. The PTV was in the range of $99.5-937.2 \mathrm{~cm}^{3}$.

\section{Treatment plan}

For treatment planning, $6 \mathrm{MV}$ and $10 \mathrm{MV}$ beams from Vital-beam linear accelerator (Varian Medical Systems, Palo Alto, California, USA) integrated with 120 millennium multi-leaf collimator (MLC) was used. Two tangential fields were applied in 3D-CRT plan with the use of more fields (where necessary) to improve the dose distribution. Field-in-fields technique with MLCs were used to reduce hot spots and the maximum dose. Tools, such as field weightings, plan normalization, and normalization at isocenter, were utilized at appropriate situations to achieve a better dose coverage. All MLCs were positioned to block a part of the lung, while the heart was considered using the beams eye view, keeping both organs at the lowest dose achievable.

The IMRT treatment plan was created with an inverse plan optimization, and the algorithm used was dose-volume optimizer. The number of fields used for plans ranged between five and seven beams with $6 \mathrm{MV}$ photon beam. The beam selection or arrangement was based on the discretion of the physicist on the best possible plan achievable. Digital reconstructed radiograph was obtained for all fields in each plan to verify the patients' position. The plans were optimized to cover the whole PTV, while sparing the organs at risk. In some cases, a $0.5 \mathrm{~mm}$ thickness of bolus was used for the optimal dose. Priority was given to the PTV and Organ at risk (OAR), which were gradually increased as deemed fit until a balance was reached between achieving good coverage and sparing OARs. The optimization process was done with the Eclipse TPS using the anisotropic analytical algorithm for calculation. The overall goal was to cover $95 \%$ of the PTV with the prescription and maximum dose below $107 \%$. The dose constraints for OAR used in this study was recommended by QUANTEC (2010) (8). For the ipsilateral lung, $10 \%$ of the total volume should not receive more than 20 Gy $(\mathrm{V} 25<10 \%$ ), while $\leq 5 \%$ of the volume of the heart should not receive more than 25 Gy (contralateral breast mean dose $\leq 3 \mathrm{~Gy}$ ).

\section{Plan evaluation}

For dosimetric analysis, the data collated from the dose volume histograms (DVHs) includes: $\mathrm{D}_{\text {near-max }}\left(D_{2}\right)$, defined as the dose delivered to $2 \%$ of the PTV; $\mathrm{D}_{\text {near-min }}\left(D_{98}\right)$, which is the dose delivered to $98 \%$ of the volume of PTV; mean dose to the PTV, $\mathrm{D}_{\text {mean }}$, and 
$\mathrm{D}_{\mathrm{p}}$, defined as the prescribed dose. Homogeneity index (HI) and conformity index (CI) were calculated according to the definition proposed by International Commission on Radiation Units and Measurements (ICRU Report 83) (9). HI and CI are estimated as:

$\mathrm{HI}=\frac{D_{2 \%-D_{98} \%}}{D_{p}}$ and $\mathrm{CI}=\frac{V(R I)}{T V}$

Where V (RI) is defined as volume of reference isodose ( $95 \%$ of the prescribed dose), while TV is the Target Volume. A more homogenous dose distribution is described by a HI value closer to zero and the closer the CI is to 1 , the higher the conformity of the dose conforms to the PTV. In other words, 0 is the ideal value for $\mathrm{HI}$ and 1 is the ideal value for $\mathrm{CI}$.

The percentage volume of the left lung receiving $\mathrm{V}_{5}$ (5 Gy), $\mathrm{V}_{10}(10$ Gy), $V_{20}(20 \mathrm{~Gy}), V_{25}(25 \mathrm{~Gy})$, and $\mathrm{D}_{\text {mean, }}$ as well as the percentage volume receiving $\mathrm{V}_{5}, \mathrm{~V}_{10}, \mathrm{~V}_{20}, \mathrm{~V}_{25}$, and $\mathrm{D}_{\text {mean }}$ to the heart were all obtained from the DVH.

\section{Statistical analysis}

All data were recorded on Microsoft Excel 2010. Statistical Package for Social Sciences for Windows, Version 18.0 (IBM Corp. Armonk, NY, USA) was used to analyze the data obtained from the DVHs. Student t-test for two independent means was used to analyze the dosimteric differences between parameters. P-value $<0.05$ was considered statistically significant.

\section{Results}

All patients IMRT plans were reviewed and approved by a radiation oncologist before treatment. A total of 20 plans for 3D-CRT and IMRT plans were created for this study, obtaining a comparison of the dosimteric parameters between both techniques. Table 1 summarizes the results of PTV in terms of $\mathrm{D}_{\text {near-max }}, \mathrm{D}_{\text {near-min }}, \mathrm{D}_{-_{\text {mean }}}, \mathrm{V}_{95}, \mathrm{HI}$, and CI.

There was a non-significant difference with IMRT compared to 3D-CRT in terms of conformity (CI) (0.994 vs $1.1586, \mathrm{p}=0.5786$ ). Similar results were seen for $\mathrm{D}_{\text {mean }}(43.20$ vs $43.13, \mathrm{p}=0.893)$ and $\mathrm{V}_{95}(567.43$ vs $518.42, \mathrm{p}=0.390)$. A huge significant difference was seen among these parameters in both techniques. However, there was significant difference between the two planning techniques in terms of $\mathrm{D}_{\text {near-max }}(44.55$ vs $46.25, \mathrm{p}=0.002), \mathrm{D}_{\text {near-min }}\left(\mathrm{D}_{98}\right)$ at $(41.73$ vs 37.71 ,

Table 1. Dosimetric comparison of PTV using 3D-CRT and IMRT $\mathrm{p}=0.001)$, and $\mathrm{HI}(0.065$ vs $0.1984, \mathrm{p}=0.0001)$. Figure $1 \mathrm{a}$ shows the $95 \%$ dose coverage and beam arrangement of 3D-CRT plans. Figure $1 \mathrm{~b}$ shows the DVH of 3D-CRT plans. Figure 2a shows the $95 \%$ dose coverage and beam arrangement of IMRT plans. Figure $2 \mathrm{~b}$ shows the DVH of IMRT plans. Regarding dose constraint to OAR, particularly the left lung and heart, immense consideration was given in all plans. All IMRT plans were clinically acceptable and fit for treatment. The left lung and heart were both within recommended constraints (QUANTEC 2010) used by the center for the IMRT plans.

Table 2 analyzes the dosimetric parameters of the OARs between the 3D-CRT plans and IMRT plans. The mean dose to the left lung was within tolerance and considered not significant between both plans (13.66 vs $18.25, \mathrm{p}=0.05$ ), while that of the Heart showed a significant difference at $(7.55$ vs $8.21, \mathrm{p}=0.7)$. There was no significant difference

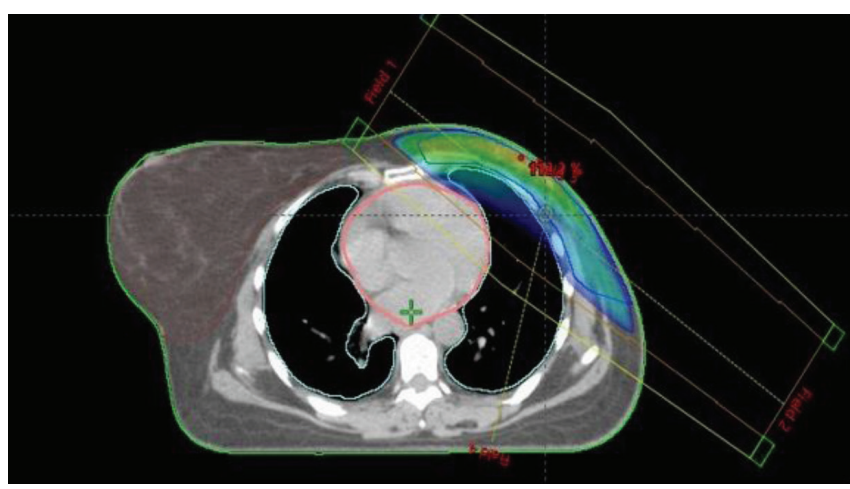

Figure 1a. PTV beam arrangement for 3D-CRT

PTV: Planning target volume; 3D-CRT: Three-dimensional conformal radiotherapy

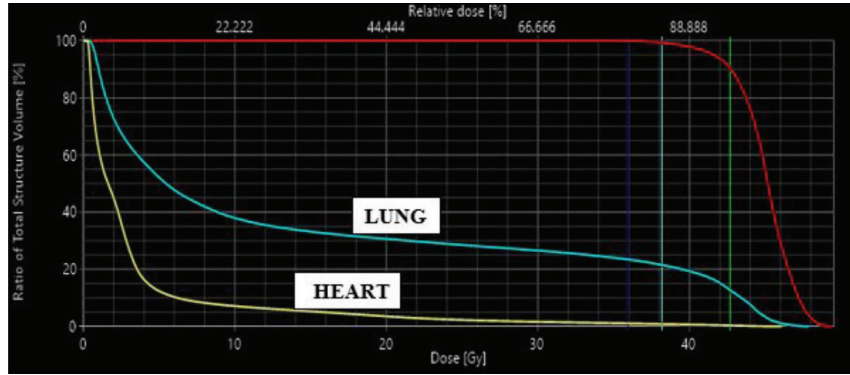

Figure 1b. PTV dose volume histogram for 3D-CRT

PTV: Planning target volume; 3D-CRT: Three-dimensional conformal radiotherapy

\begin{tabular}{lccc}
\hline Parameters & 3D-CRT & IMRT & P-value \\
\hline PTV volume $\left(\mathrm{cm}^{3}\right)$ & $569.57 \pm 227.95$ & $571 \pm 187.84$ & 0.983 \\
$\mathrm{D}_{\text {near-max }}\left(\mathrm{D}_{2}\right),(\mathrm{Gy})$ & $46.25 \pm 1.63$ & $44.55 \pm 1.52$ & 0.002 \\
$\mathrm{D}_{\text {near-min }}\left(\mathrm{D}_{98}\right),(G y)$ & $37.71 \pm 2.28$ & $41.73 \pm 1.30$ & $<0.001$ \\
Prescribed dose $\left(D_{\mathrm{p}}\right),(G y)$ & $43.05 \pm 1.49$ & $43.05 \pm 1.49$ & 1.000 \\
$\mathrm{D}_{\text {mean' }}(\mathrm{Gy})$ & $43.13 \pm 1.95$ & $43.20 \pm 1.77$ & 0.893 \\
V95, $(\%)$ & $518.42 \pm 169.30$ & $567.43 \pm 186.48$ & 0.390 \\
$\mathrm{HI}$ & $0.1984 \pm 0.065$ & $0.065 \pm 0.009$ & $<0.0001$ \\
$\mathrm{Cl}$ & $1.1586 \pm 1.314$ & $0.994 \pm 0.005$ & 0.579
\end{tabular}

PTV; $\mathrm{D}_{2}$ and $\mathrm{D}_{98}$-Dose delivered to $2 \%$ and $98 \%$ of PTV, respectively; $\mathrm{D}_{\mathrm{p}}$-Prescribed dose; $\mathrm{D}_{\text {mean }}$-Mean dose; HI: homogeneity; Cl: Conformity index; 3D-CRT: Three-dimensional conformal radiotherapy; IMRT: Intensity modulated radiotherapy techniques 


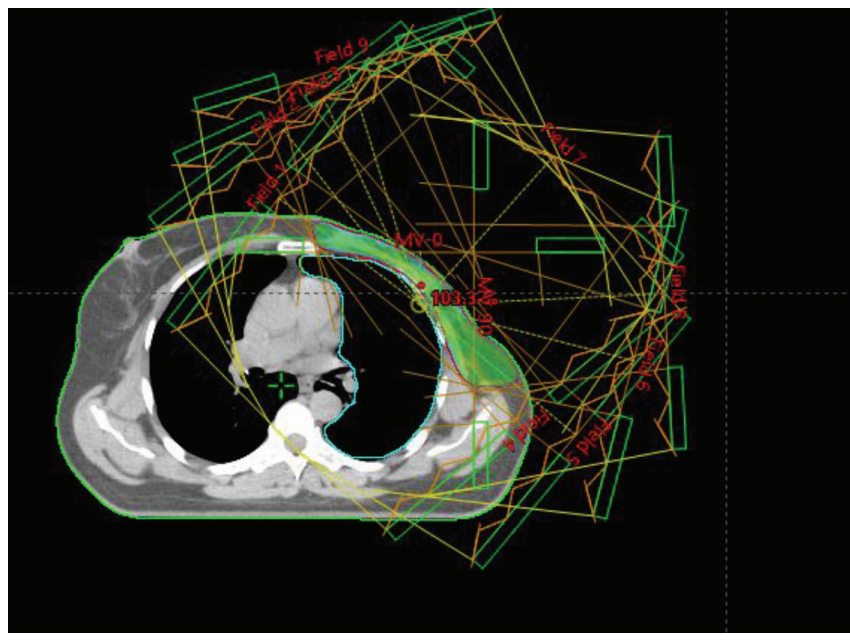

Figure 2a. PTV beam arrangement for IMRT

PTV: Planning target volume; IMRT: Intensity modulated radiotherapy techniques

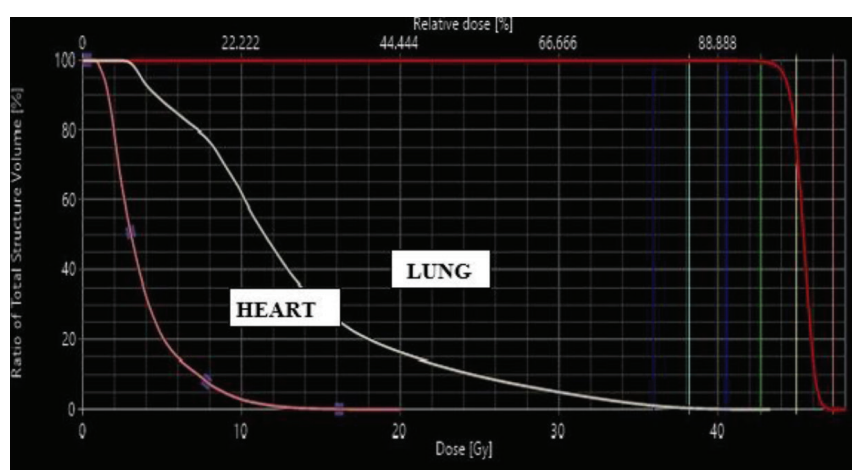

Figure 2b. PTV dose volume histogram for IMRT

PTV: Planning target volume; IMRT: Intensity modulated radiotherapy techniques in sparing of the left lung between both plans for high-dose volumes, including $\mathrm{V}_{20}$ and $\mathrm{V}_{25}$. IMRT significantly reduced the dose to the volumes $\mathrm{V}_{20}$ and $\mathrm{V}_{25}$ when compared to 3D-CRT $\left(\mathrm{V}_{20}: 18.2\right.$ vs 30.55 , $\mathrm{p}<0.0001 ; \mathrm{V}_{25}: 11.17$ vs $\left.28.12, \mathrm{p}<0.0001\right)$. For the heart, there was no significant variation, since IMRT showed supremacy to 3D-CRT at high-dose volumes $\left(\mathrm{V}_{20}: 4.44\right.$ vs $10.29, \mathrm{p}=0.02 ; \mathrm{V}_{25} .2 .08$ vs $8.94, p=0.002)$. On the other hand, 3D-CRT showed a significant exceptionality to IMRT in terms of low-dose volumes for left lung $\left(\mathrm{V}_{5}\right.$ : 92.23 vs $56.60, \mathrm{p}<0.001, \mathrm{~V}_{10} 60.98$ vs $\left.47.20, \mathrm{p}=0.04\right)$ and heart $\left(\mathrm{V}_{5}\right.$ : 57.45 vs $30.39, \mathrm{p}=0.004)$. A significant difference was seen for $\mathrm{V}_{10}$ in heart (24.48 vs 24.23, $\mathrm{p}=0.974)$ for 3D-CRT.

However, there was no significant difference between the two planning techniques in terms of $\mathrm{D}_{\text {near-max }}\left(\mathrm{D}_{2}\right)$ at $(44.55$ vs $46.25, \mathrm{p}=0.002), \mathrm{D}_{\text {near- }}$ ${ }_{\min }\left(\mathrm{D}_{98}\right)$ at $(41.73$ vs $37.71, \mathrm{p}=0.001)$ and the Homogeneity index (HI) at $(0.065$ vs $0.1984, p=0.0001)$. In comparing the dose to the contralateral breast, the mean dose and $\mathrm{V}_{5}$ were statistically significant ( 2.35 vs $1.235, \mathrm{p}=0.0016 ; 12.01$ vs $4.76, \mathrm{p}=0.008$, respectively). On the other hand, the mean dose and $\mathrm{V}_{5}$ to the contralateral lung showed no significant variation ( 4.036 vs $2.457, \mathrm{p}=0.153 ; 30.105$ vs 16.885 , $\mathrm{p}=0.127$, respectively).

\section{Discussion and Conclusion}

This study provides an optimal plan to every patient whose chest wall were radiated by IMRT, thereby protecting the OARs. Many studies have been conducted to demonstrate the superiority of one technique over another. In several trials, the advantages of IMRT over 3D-CRT with lower dose to OARs and improved dosage compliance to PTV were found in whole breast cancers. There are few accounts of radiotherapy after mastectomy (PMRT). In this study, the dose conformity, homogeneities, and sparing of OAR were analyzed for PMRT in our center. A dosimetric comparison of 3D-CRT and IMRT with left-sided mastectomy patients was done by Fiorentino et al. (10) and it was found that there was no significant difference between the two treatment planning techniques in terms of maximum dose (5.579

Table 2. Dosimetric comparison of OARs using 3D-CRT and IMRT

\begin{tabular}{|c|c|c|c|c|}
\hline Organ at risk & Parameters & 3D-CRT & IMRT & p-value \\
\hline \multirow{7}{*}{ Ipsilateral lung } & $V_{5}$ & $56.60 \% \pm 25.06 \%$ & $92.23 \% \pm 5.64 \%$ & $<0.001$ \\
\hline & $\mathrm{V}_{10}$ & $47.20 \% \pm 26.60 \%$ & $60.98 \% \pm 12.89 \%$ & 0.04 \\
\hline & $\mathrm{V}_{20}$ & $30.55 \% \pm 9.95 \%$ & $18.2 \% \pm 2.51 \%$ & $<0.0001$ \\
\hline & $\mathrm{V}_{25}$ & $28.12 \% \pm 9.21 \%$ & $11.17 \% \pm 1.96 \%$ & $<0.0001$ \\
\hline & $\mathrm{D}_{\text {mean }}(\mathrm{Gy})$ & $18.25 \pm 10.2$ & $13.66 \pm 1.29$ & 0.05 \\
\hline & $V_{5}$ & $30.39 \% \pm 33.23 \%$ & $57.45 \% \pm 20.77 \%$ & 0.004 \\
\hline & $\mathrm{V}_{10}$ & $24.23 \% \pm 31.61 \%$ & $24.48 \% \pm 13.58 \%$ & 0.9741 \\
\hline \multirow{3}{*}{ Heart } & $\mathrm{V}_{20}$ & $10.29 \% \pm 10.47 \%$ & $4.44 \% \pm 3.12 \%$ & 0.02 \\
\hline & $V_{25}$ & $8.94 \% \pm 8.98 \%$ & $2.08 \% \pm 1.56 \%$ & 0.002 \\
\hline & $\mathrm{D}_{\text {mean }}(\mathrm{Gy})$ & $8.21 \pm 7.66$ & $7.55 \pm 2.25$ & 0.7 \\
\hline \multirow{3}{*}{ Contralateral breast } & $\mathrm{D}_{\text {mean }}(\mathrm{Gy})$ & $1.234 \pm 1.069$ & $2.35 \pm 1.004$ & 0.0016 \\
\hline & $\mathrm{V}_{5}$ & $4.76 \pm 4.98$ & $12.01 \pm 10.63$ & 0.008 \\
\hline & $\mathrm{d}-_{\text {mean }}(\mathrm{Gy})$ & $2.457 \pm 4.614$ & $4.036 \pm 1.479$ & 0.153 \\
\hline Contralateral lung & $\mathrm{V}_{5}$ & $16.885 \pm 33.766$ & $30.105 \pm 17.4$ & 0.127 \\
\hline
\end{tabular}

$\mathrm{D}_{\text {mean }}$ : Mean dose; $\mathrm{V}_{\mathrm{x}}$ : Volume of tissue receiving $\mathrm{X}$ Gy; 3D-CRT: Three-dimensional conformal radiotherapy; IMRT: Intensity modulated radiotherapy techniques; OAR: Organ at risk 
vs $5.529, \mathrm{p}=0.51)$, minimum dose (3.900 vs 3.887, $\mathrm{p}=0.85)$, mean dose (4.698 vs 5.137, p = 0.33), and homogeneity index (1.17 vs 1.16, $\mathrm{p}=0.47$ ). Recently, Shanei et al. (11) conducted a radiobiological comparison of 3D-CRT and IMRT in left-sided radiotherapy breast cancer. Their results showed a significant increase in mean dose of the target $(\mathrm{p}<0.001)$ and a significant difference in CI and HI for six to nine fields IMRT plans than 3D-CRT at $\mathrm{p}<0.001$. In the current study, there was a non-significant difference in mean dose to the target $(\mathrm{p}=0.983)$ and CI $(\mathrm{p}=0.5786)$, with HI significant at $(\mathrm{p}<0.001)$. In 2013, Moorthy et al. (12) compared the dosimetric properties of SIB-IMRT and SIB-3D-CRT for breast cancer using breath-holding gated technique. Although no significant difference was seen in HI ( $\mathrm{p}$ $=0.45)$ for both techniques, an improvement in CI from 3D-CRT (0.18) to IMRT $(0.14)$ at ( $\mathrm{p}=0.01)$ was observed. Also, it was stated that IMRT reduced the dose to the OAR better than 3D-CRT. In a research study regarding PMRT in 3D-CRT and IMRT, Rastogi et al. (13) concluded that IMRT significantly improves CI at $\mathrm{p}<0.001$ and that no significant difference was seen in both techniques for mean dose, although IMRT, in comparison with 3D-CRT, significantly reduced the high-dose volumes of ipsilateral lung and heart, 3D-CRT is superior in low-dose volume. Li et al. (14) also investigated IMRT and 3D-CRT in post-mastectomy irradiation of chest wall and regional nodes and found that low-dose volumes to the ipsilateral lung were better spared in 3D-CRT, while high-dose volumes to the lungs were better spared in IMRT; and homogeneity and conformity were also better in IMRT than in 3D-CRT. Findings from these studies are similar to those of our study for HI, which showed a significant variation, the current $\mathrm{CI}$ is considered non-significant. It is worthy to state that the heterogenous dose distribution, hot/cold spots due to irregular breast contour, difficulty in establishing dose consistency, and homogeneity are few of the challenges found in 3D-CRT technique. Normal tissues are exposed to radiation during treatment of primary cancer. The dose to the contralateral breast and lung has been proven to cause secondary cancer. The IMRT treatment plans adhered to the QUANTEC constraint of less than 3 Gy for contralateral breast. A significant difference was seen in mean dose and $V_{5}$ for contralateral breast $(\mathrm{p}=0.0016)$. This was in line with the results reported in Serhat et al. (15).

Radiation pneumonitis and cardiac morbidity are major concerns in patients who have received radiotherapy after a mastectomy. Many factors, including radiation fractionation, volume of lung radiated, intake of chemotherapy drugs, and increasing use of CT, have been implicated in the increasing rate of this complication $(16,17)$. Although there is no threshold or safe dose to which they do not occur, dose to the lungs and heart should be kept as low as achievable and this is seen in the present study. IMRT showed better sparing in $\mathrm{V}_{20}$ and $\mathrm{V}_{25}$ to the left lung and heart. The geometric difference between whole breast cancers and PMRT (chest wall radiation) may have an impact on the dose distribution; in other words, the anatomical structure of the whole breast and chest wall are much different. This may be a reason for the differences in dose distribution to OAR found in other studies. We encountered a few limitations during the research, one of which was the less number of patients. Another was a short follow-up on radiation toxicity and local control in IMRT plans, which was not addressed in this work. In the future, further studies are encouraged to address these limitations.

In conclusion, this work showed better homogeneity and conformity of dose to target volume in IMRT than in 3D-CRT. However, the 3D-CRT was found to be exceptional in low-dose volumes for left lung and heart $\left(\mathrm{V}_{5}\right.$ and $\left.\mathrm{V}_{10}\right)$ than IMRT. IMRT showed superiority in high-dose volumes $\left(\mathrm{V}_{20}\right.$ and $\left.\mathrm{V}_{25}\right)$ to the organ at risk. IMRT was proven to be a better technique than 3D-CRT in terms of dose homogeneity and sparing of OAR, however, no substantial difference was seen in CI.

\section{Acknowledgements}

We want to appreciate Abdallah E Kotkat, Ibrahim EL Hamamsi, Mr Lawal Rasak, Dr. Tolu Adewole, Prof. Fasanmade, Prof. Durosinnmi-Etti and Prof. Aweda for their great contribution towards the success of this work.

Ethics Committee Approval: ADM/DCST/HREC/APP/3783, 13/08/2020, Lagos University Teaching Hospital Health Research Ethics Committee.

Informed Consent: It was taken.

Peer-review: Externally peer-reviewed.

\section{Authorship Contributions}

Conception: S.A., B.A.; Design: S.A., M.A., B.A.; Supervision: M.A., B.A.; Materials: A.A., N.A.; Data Collection and/or Processing: A.A., E.O.; Analysis and/or Interpretation: N.A., O.F.; Literature Review: A.J., A.O.; Writing: S.A., N.A. Ad.A; Critical Review: A.A., O.F.

Conflict of Interest: No conflict of interest was declared by the authors.

Financial Disclosure: The authors declared that this study received no financial support.

\section{References}

1. Youlden D, Cramb S, Yip CH, Baade P. Incidence and mortality of female breast cancer in the Asia-Pacific region. Cancer Biol Med 2014; 11: 101115. (PMID: 25009752) [Crossref]

2. National Cancer Institute. SEER-Medicare: Brief description of the SEER-Medicare database. Last Accessed Date: 10.06.2020. Available from: http://healthservices.cancer.gov/seermedicare/overview/brief.html [Crossref]

3. Awofeso O, Roberts AA, Salako O, Balogun L and Okediji P. Prevalence and pattern of late-stage presentation in women with breast and cervical cancers in Lagos University Teaching Hospital, Nigeria. Niger Med J 2018; 59: 74-79. (PMID: 31423046) [Crossref]

4. Darby S, McGale P, Correa C, Taylor C, Arriagada R, Clarke M, et al. Effect of radiotherapy after breast-conserving surgery on 10-year recurrence and 15-year breast cancer death; meta-analysis of individual patient data for 10,801 women in 17 randomized trials. Lancet 2011; 378: 771-784. (PMID: 22019144) [Crossref]

5. Clarke M, Collins R, Darby S, Davies C, Elphinstone P, Evans V, et al. Effect of radiotherapy and of differences in the extent of surgery for early breast cancer on local recurrence and 15-year survival: an overview of the randomized trials. Lancet 2005; 366: 2087-2106. (PMID: 16360786) [Crossref]

6. Rastogi K, Sharma S, Gupta S, Agarwal N, Bhaskar S, Jain S. Dosimetric comparison of IMRT versus 3DCRT for post-mastectomy chest wall irradiation. Radiat Oncol J 2018; 36: 71-78. (PMID: 29621872) [Crossref]

7. 2018 Varian Eclipse Plan Challenge Aims to Improve Lung SBRT Plan Quality, 2018. Last Accessed Date: 11.12.2020. Available from: https:// www.varian.com/resources-support/blogs/clinical-oncology-news/2018varian-eclipse-plan-challenge-aims-improve-lung [Crossref]

8. Bentzen SM, Constine LS, Deasy JO, Eisbruch A, Jackson A, Marks LB, et al. Quantitative Analyses of Normal Tissue Effects in the Clinic 
(QUANTEC): an introduction to the scientific issues. Int J Radiat Oncol Biol Phys 2010; 76(Suppl 3): S3-S9. doi: 10.1016/j.ijrobp.2009.09.040 (PMID: 20171515) [Crossref]

9. Menzel HG. International Commission of Radiological Units (ICRU) Report 83. J ICRU. 2010; 10: Report 83 Oxford University.

10. Fiorentino A, Ruggieri R, Giaj-Levra N, Sicignano G, Di Paola G, Naccarato $S$, et al. Three-dimensional conformal versus intensity modulated radiotherapy in breast cancer treatment: Is necessary a medical reversal? Radiol Med 2017; 122: 146-153. (PMID: 27778239). [Crossref]

11. Shanei A, Amouheidari A, Abedi I, Kazemzadeh A, Jaafari A. Radiobiological comparison of 3D conformal and intensity modulated radiation therapy in the treatment of left-sided breast cancer. Int J Radiat Res 2020; 18: 315-322. [Crossref]

12. Moorthy S, Sakr H, Hasan S, Samuel J, Al-Janahi S, Murthy N. Dosimetric study of SIB-IMRT versus SIB-3DCRT for breast cancer with breath-hold gated technique. Int J Cancer Ther Oncol 2013; 1: 010110. doi:10.14319/ijcto.0101.10 [Crossref]

13. Rastogi K, Sharma S, Gupta S, Agarwal N, Bhaskar S, Jain S. Dosimetric comparison of IMRT versus 3DCRT for post-mastectomy chest wall irradiation. Radiat Oncol J 2018; 36: 71-78. (PMID: 29621872) [Crossref]

14. Li W, Ma J, Chen J, Zhang Z. IMRT versus 3D-CRT for postmastectomy irradiation of chest wall and regional nodes: a population-based comparison of normal lung dose. Int J Radiat Oncol Biol Phys 2014; 1: S246-S247. [Crossref]

15. Serhat A, Turkan I, Meryem A. Dosimetric comparison of ThreeDimensional Conformal Radiotherapy (3D-CRT) and Intensity Modulated Radiotherapy Techniques (IMRT) with radiotherapy dose simulations for left-sided mastectomy patients. Eur J Breast Health 2019; 15: 85-89. (PMID: 31001609) [Crossref]

16. Lingos T, Recht A, Vicini F, Abner A, Silver B, Harris JR. Radiation pneumonitis in breast cancer patients treated with conservative surgery and radiation therapy. Int J Radiat Oncol Biol Phys 1991; 21: 355-360. (PMID: 2061112) [Crossref]

17. Travis E. Lung morbidity of radiotherapy. In: Plowman P, McElwain TJ, Meadows AT, editors. Complications of cancer management. Oxford (UK): Butterworth-Heineman; 1991. p. 232-249. [Crossref] 